\title{
छs \\ Mapping spin-polarized transitions with atomic resolution
}

\author{
P. Schattschneider, ${ }^{1,2}$ B. Schaffer, ${ }^{3,4}$ I. Ennen, ${ }^{1}$ and J. Verbeeck ${ }^{5}$ \\ ${ }^{1}$ Institut für Festkörperphysik, Technische Universität Wien, A-1040 Wien, Austria \\ ${ }^{2}$ University Service Centre for Electron Microscopy, Technische Universität Wien, A-1040 WIEN, Austria \\ ${ }^{3}$ SuperSTEM, STFC Daresbury Laboratories, Keckwick Lane, Warrington, WA4 4AD, United Kingdom \\ ${ }^{4}$ Kelvin Nanocharacterisation Centre, SUPA School of Physics and Astronomy, University of Glasgow, Glasgow, \\ G12 8QQ, Scotland, United Kingdom \\ ${ }^{5}$ EMAT, University of Antwerp, Groenenborgerlaan 171, 2020 Antwerp, Belgium
}

(Received 22 November 2011; published 11 April 2012)

\begin{abstract}
The coupling of angstrom-sized electron probes with spin-polarized electronic transitions shows that the inelastically scattered probe electron is in a mixed state containing electron vortices with nonzero orbital angular momentum. These electrons create an asymmetric intensity distribution in energy filtered diffraction patterns, giving access to maps of the magnetic moments with atomic resolution. A feasibility experiment shows evidence of the predicted effect. Potential applications are column-by-column maps of magnetic ordering, and the creation of angstrom-sized free electrons with orbital angular momentum by inelastic scattering in a thin ferromagnetic foil.
\end{abstract}

DOI: $10.1103 /$ PhysRevB.85.134422

PACS number(s): 75.25.-j, 03.65.Vf, 82.80.Dx, 78.20.Fm

\section{INTRODUCTION}

With the availablity of electron vortices of sub-nanometer scale in the transmission electron microscope ${ }^{1-4}$ (TEM) and its theoretical description, ${ }^{5-7}$ many potential applications come within reach, ranging from the transfer of angular momentum to nanoparticles, over utilization of the intrinsic magnetic moment of vortex electrons to the probing of chirality. ${ }^{8}$ Indeed, chiral electronic transitions were the first application of electron vortices in energy-loss magnetic chiral dichroism (EMCD). ${ }^{1}$

The discovery of EMCD (Ref. 9) was an unexpected alternative to XMCD (x-ray magnetic circular dichroism) with the convenient side effect that additional information on the investigated material can be obtained simultaneously via standard analytical techniques. ${ }^{10,11}$ The spatial resolution of this electron-microscopic technique is now in the nanometer range. ${ }^{12} \mathrm{~A}$ modification of the technique has been shown to be site selective. ${ }^{13}$ The excellent spatial resolution and the site selectivity are important for the study of novel materials such as Heusler alloys, ${ }^{14,15}$ nanoparticles, or interfaces. ${ }^{16}$ Recent advances in electron microscopy have led to the imaging of condensed matter with subatomic resolution. ${ }^{17-19}$ On this basis, it has been speculated that the mapping of spin-polarized electronic transitions, and thus the mapping of spin and orbital polarization, on the atomic scale could be feasible in a TEM, applying the EMCD technique. ${ }^{20}$ An incident plane wave affecting a spin-polarized $L_{23}$ transition would break the mirror symmetry of nonmagnetic transitions in the scattered wave. This symmetry breaking could be analyzed either with asymmetric objective apertures or with a cylinder lens. ${ }^{21,22}$ However, for technical reasons, both approaches are unrealistic in the TEM.

On second thought, it becomes evident that the inelastic interaction of an incident electron with a spin-polarized electronic transition creates a scattered electron with topological charge. One can thus apply the theory of vortex electrons ${ }^{6}$ to the outgoing wave field. In a sense, this is a bottom-up application of the original idea of using incident vortex electrons for EMCD. The reason that this works is the generalized reciprocity theorem ${ }^{23}$ that confirms the equivalence of the incident and the outgoing (also called reciprocal ${ }^{24}$ ) electron for inelastic scattering. This observation raises two questions: How can an EMCD signal be detected with angstrom-sized scanning TEM (STEM) probes? Can one produce electron vortices without holographic masks?

Here, we present a theoretical and numerical analysis of the coupling between an angstrom-sized STEM probe and an atom-sized vortex field via a chiral electronic transition. It is shown that the inelastically scattered probe is in a mixed state containing electron vortices with nonzero angular momentum. These electrons create an asymmetric intensity distribution in energy filtered diffraction patterns, giving access to maps of the magnetic moments on an atomic column-by-column basis. A feasibility experiment shows evidence of the predicted effect. Finally, potential applications are discussed: maps of magnetic ordering with atomic resolution, and the creation of free electrons with orbital angular momentum and a diameter of about $0.1 \mathrm{~nm}$ by inelastic scattering in a thin ferromagnetic foil.

\section{THEORY}

We focus on the model of a thin (ideally one atom) layer of Fe. In this case, the dynamical equation for the propagation of the probe's density matrix is considerably simplified. We give here only the basic equation for the propagator and refer the reader to the relevant literature..$^{20,22,25-27}$

The inelastic intensity at energy loss $E$ in the exit plane of the specimen is the diagonal term $\rho(\mathbf{r}, \mathbf{r})$ of the density matrix:

$$
\begin{aligned}
\rho_{E}\left(\mathbf{r}, \mathbf{r}^{\prime}\right)= & \iint G_{d-z}(\mathbf{r}, \mathbf{x}) G_{d-z}^{*}\left(\mathbf{r}^{\prime}, \mathbf{x}^{\prime}\right) T_{E}\left(\mathbf{x}, \mathbf{x}^{\prime}, z, z^{\prime}\right) \\
& \times \phi_{z}^{*}(\mathbf{x}) \phi_{z^{\prime}}\left(\mathbf{x}^{\prime}\right) d^{2} x d^{2} x^{\prime} e^{i q_{e}\left(z-z^{\prime}\right)} d z d z^{\prime},
\end{aligned}
$$

where $z, z^{\prime}$ are variables along the optic axis and $\mathbf{r}, \mathbf{x}$ are in planes perpendicular to the optic axis. $G$ is the elastic propagator of electrons in the crystal. $\phi_{z}$ is the wave function 
of the incident electron at depth $z$ and $q_{E}=k_{o}-k_{i}$ is the minimum wave-number transfer in the inelastic interaction, equal to the difference between wave numbers of the outgoing and the incident electrons.

The inelastic scattering kernel $T_{E}\left(\mathbf{x}, \mathbf{x}^{\prime}, z, z^{\prime}\right)$ is characteristic for the electronic transitions creating an energy loss $E$. For thin specimens and atomic columns without defects, the $z$ integration can be performed in closed form. ${ }^{28}$ For spinpolarized dipole transitions, the atomic scattering kernel reads $\mathrm{as}^{22}$

$$
T_{E}(\mathbf{x}, \mathbf{x})=\sum_{\mu=-1}^{1}\left|\psi_{\mu}(\mathbf{x})\right|^{2} \sum_{s=\uparrow, \downarrow} C_{j \mu}^{\uparrow, \downarrow} n^{\uparrow, \downarrow}
$$

with

$$
\begin{gathered}
\psi_{ \pm 1}(\mathbf{x})=e^{ \pm i \alpha} \frac{i}{2 \pi} \int_{0}^{\infty} \frac{q^{2} J_{1}(q x)\left\langle j_{1}(Q)\right\rangle_{E L S j}}{Q^{3}} d q, \\
\psi_{0}(\mathbf{x})=\frac{q_{E}}{2 \pi} \int_{0}^{\infty} \frac{q J_{0}(q x)\left\langle j_{1}(Q)\right\rangle_{E L S j}}{Q^{3}} d q,
\end{gathered}
$$

with the matrix element of the spherical Bessel function

$$
\left\langle j_{1}(Q)\right\rangle=\left\langle I\left|j_{1}(Q)\right| F\right\rangle
$$

between initial and final target states. $Q^{2}=q^{2}+q_{E}^{2}$ with the characteristic wave-number transfer $q_{E}=k_{0} E /\left(2 E_{0}\right)$. The coefficients $C_{j \mu}$ are weighting factors for spin-orbit coupling, ${ }^{22,29,30}$ and $n^{\uparrow, \downarrow}$ is the spin polarization of the final state. The spin-orbit coupling of the initial state renders the coefficients $C$ dependent on the total magnetic quantum number $j=l+s$. For the $L_{23}$ edges to be considered, $j=1 / 2$ or $3 / 2$. The essential property that we will focus on is described by Eq. (3a): It represents the outgoing inelastically scattered wave as an electron vortex with topology $m= \pm 1$ in the form of a Hankel transform that is easily computable from atomic wave functions. The azimuthal phase factor $e^{ \pm i \alpha}$ shows that the outgoing probe electron has orbital angular momentum. It should be mentioned that the probe beam is not spin polarized. It has acquired orbital angular momentum by spin-orbit coupling of the target electrons.

The propagation of focused probes through a thin specimen has regained interest in the context of real-space STEM. ${ }^{31}$ Even for elastic scattering, the problem of propagating a focused probe, as we shall adopt in the following, through a thin specimen to the detector poses considerable numerical problems. The inelastic interaction that can take place throughout the specimen adds another complexity. Therefore, Eq. (1) can not be solved without approximations, at least with present numerical capacity. We restrict the discussion to a model system that allows us to analyze the salient features of the inelastic coupling process with an accuracy comparable to the available experimental data. As such, we choose a line of equally spaced atoms with given spin polarization; we shall calculate the contributions from each transition in dipole approximation, discuss the signal from a single atom, and finally build a line profile of the energy filtered signal from the array of atoms. For this model, the elastic propagators $G$ in Eq. (1) collapse into delta functions and we have for the diagonal element (the density)

$$
\rho_{E}(\mathbf{r}, \mathbf{r})=T(\mathbf{r}, \mathbf{r}) \phi_{0}^{*}(\mathbf{r}) \phi_{0}(\mathbf{r}) .
$$

Equation (2) allows us to disentangle the problem: Each transition channel $\mu \in[-1,1]$ can be factorized into a product of wave functions, and we get for the outgoing intensity in channel $\mu$

$$
\rho_{j \mu}(\mathbf{r}, \mathbf{r})=\bar{C}_{j \mu} \varphi_{\mu}(\mathbf{r}) \varphi_{\mu}^{*}(\mathbf{r})
$$

with

$$
\bar{C}_{j \mu}=\sum_{s=\uparrow, \downarrow} C_{j \mu}^{\uparrow, \downarrow} n^{\uparrow, \downarrow}
$$

and

$$
\varphi_{\mu}(\mathbf{r})=\psi_{\mu}(\mathbf{r}) \phi_{i}^{*}(\mathbf{r}) .
$$

The intensity in the diffraction plane is calculated from the two-dimensional (2D) Fourier transform of Eq. (5):

$$
\rho_{j \mu}(\mathbf{q}, \mathbf{q})=\bar{C}_{j \mu}\left|F T_{\mathbf{r}}\left[\varphi_{\mu}(\mathbf{r})\right]\right|^{2}
$$

as the trace over the three transition channels $\mu$ :

$$
\rho_{j, E}(\mathbf{q}, \mathbf{q})=\operatorname{Tr}_{\mu}\left[\rho_{j \mu}(\mathbf{q}, \mathbf{q})\right]=\sum_{\mu} \rho_{j \mu}(\mathbf{q}, \mathbf{q}) .
$$

The trace operator shows formally that the scattered electron is in a mixed state. With the coefficients $C$ for $L_{23}$ transitions, we can compute the outgoing intensity and the corresponding diffraction patterns. Without loss of generality, we assume complete spin polarization for the final target states (as is justified for the $L$ edges of the $3 d$ ferromagnets that we will use as a demonstration example).

For complete spin polarization, the values for $\bar{C}_{j \mu}$ are given in Table I. ${ }^{22}$ The image intensity Eq. (4) will then contain different contributions from the scattering channels for spin-up and-down polarizations. This difference is the basis of EMCD.

From here on, we focus on the $L_{3}$ edge and omit the index $j$ for easier readability. By scrutinizing Eq. (5) for an incident plane wave, one notes that $\left|\varphi_{\mu}\right|^{2}=\left|\varphi_{-\mu}\right|^{2}$ because the phase factors of both the incident plane wave and the kernel $e^{ \pm i \alpha}$ cancel in the intensity. That means that there will be no difference in the image predicted by Eq. (5) for spin-up or for spin-down polarizations. The same is true for the intensity in the diffraction plane Eq. (9). Essentially, it is not possible to see spin polarization from single atoms in the TEM without further action. (In the standard EMCD geometry, one uses the interference terms caused by Bragg scattering of the outgoing atomic vortices on the lattice, which is different for spin-up and -down polarizations.)

The situation changes when using a STEM probe instead of a plane incident wave. We analyze the situation qualitatively before considering numerical simulations. For the explanation of the effect, we assume a narrow focused probe given by the Airy function $\mathcal{A}$ with a diameter much smaller than the distance $\mathbf{R}$ from the atom, essentially so small that that the amplitude of the kernel is almost constant within the probe. (In the numerical simulation, this condition is relieved.) Then, we can approximate the outgoing wave in channel $\mu$ as

$$
\varphi_{\mu}(\mathbf{r})=\psi_{\mu}(\mathbf{r}+\mathbf{R}) \mathcal{A}(\mathbf{r}) \approx \mathcal{A}(\mathbf{r})\left|\psi_{\mu}(R)\right| e^{i \mu \alpha(\mathbf{r}+\mathbf{R})} .
$$

Note that the outgoing wave is more extended than the Airy disk because of the long-range Coulomb coupling force; in Fig. 1, it is drawn not to scale (even larger for better visibility). 
TABLE I. Prefactors $C_{j \mu}^{\uparrow, \downarrow}$. The first two rows are the weighting factors for the transitions when the final states are completely (up- or down-) spin polarized. The third row gives the weighting factors for unpolarized final states (all per electron).

\begin{tabular}{lccccc}
\hline \hline$j$ & \multicolumn{1}{c}{$1 / 2\left(L_{2}\right)$} & & $3 / 2\left(L_{3}\right)$ \\
\hline$\mu$ & -1 & 0 & 1 & -1 & 0 \\
$\uparrow$ & 0.056 & 0.111 & 0.167 & 0.278 & 0.222 \\
$\downarrow$ & 0.167 & 0.111 & 0.056 & 0.167 & 0.167 \\
Unpolarized & 0.111 & 0.111 & 0.111 & 0.222 & 0.278 \\
\hline \hline
\end{tabular}

The azimuth angle within the outgoing disk is $\alpha \doteq \alpha(\mathbf{R})+$ $\mu y / R$ in the coordinate system shown in Fig. 1, and we see that the phase of the outgoing wave depends on the position of the STEM probe and changes sign when going from $\mu=$ 1 to -1 . Via the shift theorem, the diffraction pattern will be proportional to the Fourier transform of the Airy function shifted in $q_{y}$ direction by $-1 / R, 0,1 / R$ for the three transition channels, which are shifted disk functions $\Pi(\mathbf{q})$ :

$$
\rho_{\mu}(\mathbf{q}, \mathbf{q})=\bar{C}_{j \mu}\left|\psi_{\mu}(R)\right|^{2} \Pi\left(\mathbf{q}+\mu \hat{\mathbf{q}}_{y} / R\right) .
$$

Having established an observable that has the signature of a particular transition channel, the spin polarization of a single atomic column can be determined.

\section{NUMERICAL SIMULATIONS}

Simulations were performed for an incident probe of $100 \mathrm{keV}$. First, we construct the outgoing signal for each of the three transition channels $\rho_{\mu}(\mathbf{r}, \mathbf{r})$ shown in Fig. 2 . These are $L_{3}$ energy filtered images of a STEM probe of $0.1 \mathrm{~nm}$ diameter scanning across a single atom. From top to bottom are the transition channels $\mu=-1,0,+1$. From left to right the distance $R$ to the atom is $-2,-1,0,1,2$ atomic units. Note (a)

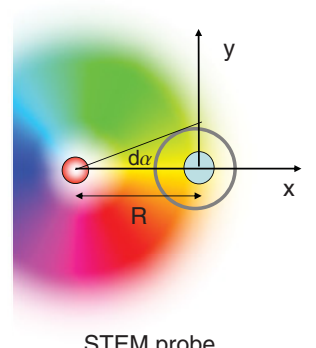

STEM probe (b)

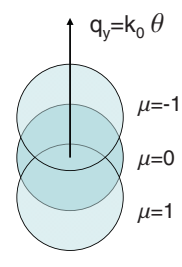

diffraction pattern
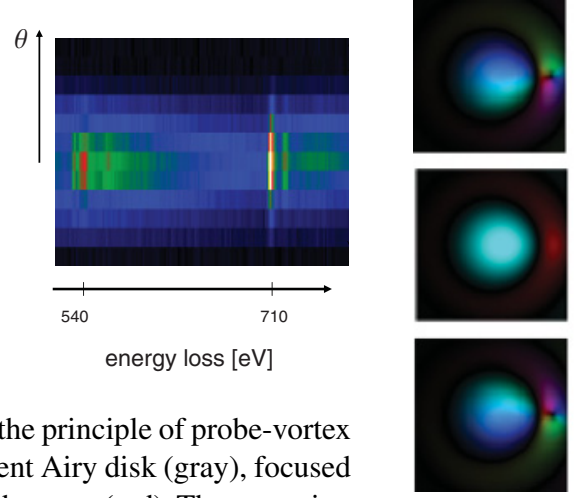
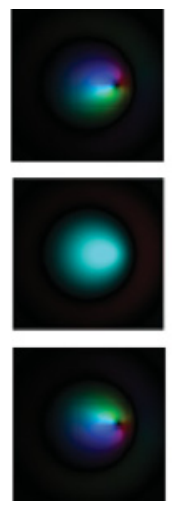
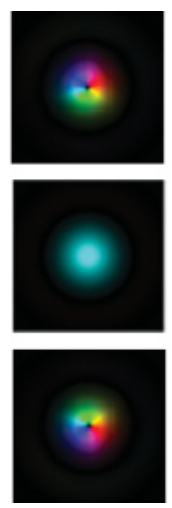
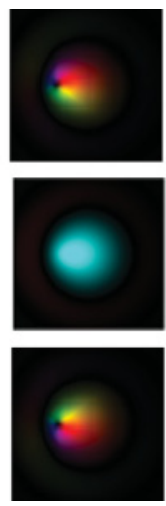
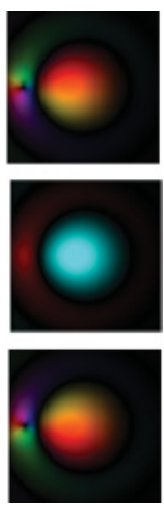

coupling. (a) Top view of a narrow incident Airy disk (gray), focused at a distance $R$ in scan direction ( $x$ ) from the atom (red). The scattering kernel $T_{E}$ is symbolized as a diffuse cloud with a color-coded phase (hereafter called rainbow wheel), increasing from blue to red in clockwise rotation for the $\mu=1$ channel. According to Eq. (10), the outgoing wave (gray circle) has acquired a phase ramp $d \alpha=\mu y / R$ in the chiral transition. (b) The phase ramp translates into a shift of the diffraction disk by $\mu / R$ in direction $q_{y}$. (c) The $q_{x}$ extension is squeezed into one pixel on the detector in the $(q, E)$ geometry. Note that the energy-loss axis must be perpendicular to $q_{y}$, which is proportional to the scattering angle $q_{y}=k_{0} \theta$. The $(\theta, E)$ map is a true image taken on magnetite ranging from the oxygen $K$ edge at $\sim 540 \mathrm{eV}$ to the $\mathrm{Fe} L_{23}$ edge at $\sim 710 \mathrm{eV}$.

FIG. 2. (Color online) $\mathrm{Fe} L_{3}$ energy filtered real-space exit wave functions $\varphi_{\mu}$ [Eq. (7)] of a 100-keV STEM probe of $0.1 \mathrm{~nm}$ diameter scanning across a single atom for the three dipole allowed transition channels. From top to bottom: transition channels $\mu=-1,0,+1$. From left to right: distance $R$ to the atom $-2,-1,0,1,2$ atomic units. Brightness codes are for intensity of the image, and color codes for the phase of the wave function (rainbow wheel as given in Fig. 1). When the Airy disk sits on the atom (center column), the outgoing beam is a real vortex with $\mu \in[-1,0,1]$. At a distance, phase ramps in the Airy disks develop, changing sign with $R$ and with $\mu$. Each square has a side length of 5 atomic units $(\sim 0.26 \mathrm{~nm})$. 

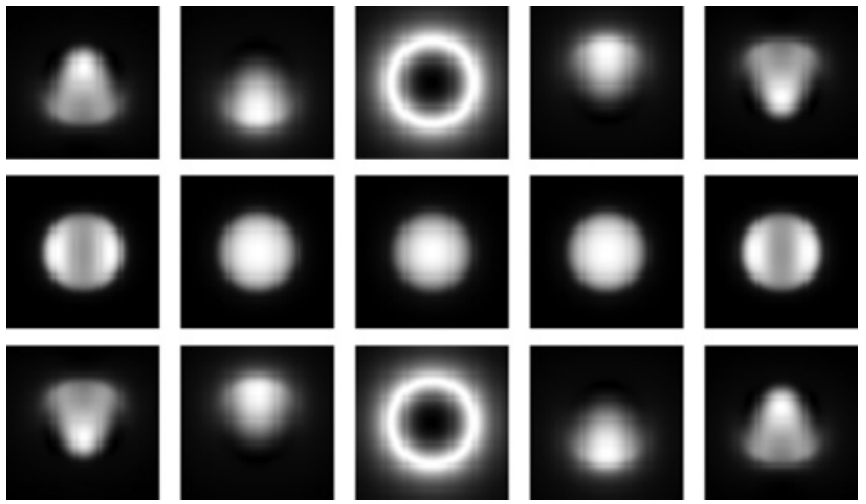

FIG. 3. Fe $L_{3}$ energy filtered diffraction patterns $\rho(\mathbf{q}, \mathbf{q})_{\mu}$ [Eq. (8)] of a 100-keV STEM probe of $0.1 \mathrm{~nm}$ diameter scanning across a single atom, corresponding to Fig. 2. From top to bottom: transition channels $\mu=-1,0,+1$. From left to right: distance $R$ to the atom $-2,-1,0,1,2$ atomic units. Note the shift of the patterns for $\mu= \pm 1$ in vertical direction $q_{y}$ as predicted in Fig. 1(b), depending on the position of the probe, and the inversion of shifts with change of sign. The $\mu=0$ channel does not show any shift because there is no phase ramp in the image. Convergence angle $18 \mathrm{mrad}$. The images have a side length of $\pm 50 \mathrm{mrad}$.

there, the difference is only in the phase, not in the intensity distribution.)

Monitoring energy filtered diffraction patterns of a scanned probe means measuring a multidimensional data cube because background subtraction and multiple scattering deconvolution of energy-loss spectra require a range of losses. Such data have two dimensions $\left(q_{x}, q_{y}\right)$ in the reciprocal space, one in the energy-loss $(\mathrm{eV})$, and one $(x)$ or two $(x, y)$ in the real space, depending on the scanning pattern. This creates huge data files (a scan over 1 elementary cell in magnetite with 0.02 -nm step width with $256^{2}$ pixels in the diffraction pattern would give $\sim 60 \mathrm{Mb}$ per energy channel, approaching $\sim 10 \mathrm{~GB}$ for a whole spectrum) and is impractical. One can, however, exploit a remarkable feature apparent in the simulations: The diffraction patterns show only asymmetry with respect to the coordinate $q_{y}$, which is the Fourier-transformed variable of $y$. An experimental setup could then discard the $q_{x}$ variable by integration without information loss. This is exactly what is realized in the $(q, E)$ geometry. There, the $q_{x}$ axis is squeezed onto one pixel of the spectrometer by compressive lenses, whereas the $q_{y}$ axis is retained, being projected on the detector perpendicular to the energy-loss axis. This is sketched in Fig. 1(c), which is a $\left(q_{y}, E\right)$ data set for a fixed position $R$. Selecting the $L_{3}$ white line from the whole $\left(q_{y}, E, R\right)$ data, a reduced subset with axes $\left(R, q_{y}\right)$ is obtained. The scan direction must be perpendicular to $y$ in the coordinate system of Fig. 1 . Contrary to XMCD or EMCD, the new technique operates on a single white line only (the stronger $L_{3}$ line here). This is important because often the $L_{2}$ edge is too faint to obtain sensible results. (It should be noted that for the separation of spin and orbital moments, both $L_{2}$ and $L_{3}$ edges are needed. ${ }^{32,33}$ )

These subsets can be constructed from the previous results, simply by integrating the intensity of Fig. 3 over the "squeezed" variable $q_{x}$ and summing over the three transition channels in Eq. (9). The result is shown in Fig. 4 for spin-up
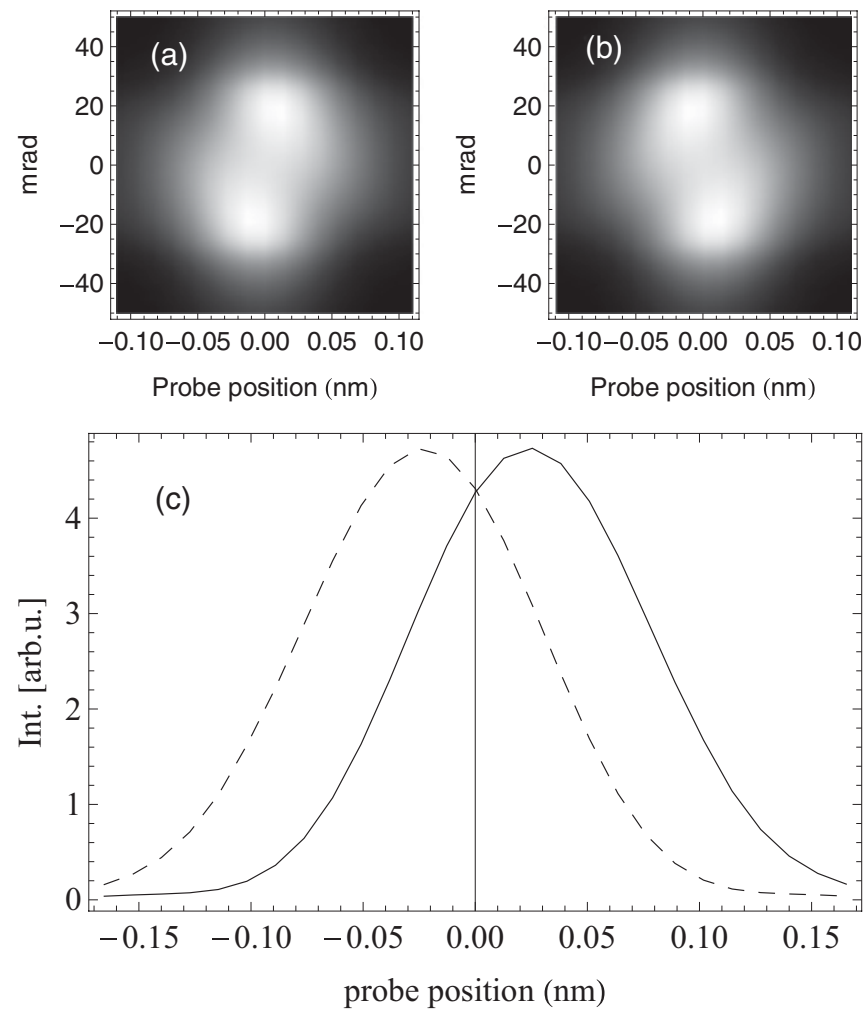

FIG. 4. Fe $L_{3}$ energy filtered $\left(R, q_{y}\right)$ signal distribution of a STEM probe of $0.1 \mathrm{~nm}$ diameter scanning across a single atom, corresponding to Fig. 2. (a) Spin-up polarization of the atom; (b) spin-down polarization. The top/bottom asymmetry is well visible and can be used to determine the spin polarization. (c) Full line: intensity integrated over the upper detector half (20 to $50 \mathrm{mrad}$ ) line scan of a STEM probe of $0.1 \mathrm{~nm}$ diameter across a single atom, corresponding to (a). Dashed line: same for the lower detector half ( -50 to $-20 \mathrm{mrad}$ ).

and -down configurations. The asymmetry of the intensity distribution with respect to the atom position at $R=0$ is indicative for spin-polarized transitions.

To enhance the asymmetry, Fig. 4 suggests to avoid the central part around $q_{y}=0$, which adds only spin-insensitive intensity, thus increasing the noise level. Integration of the density matrix Eq. (8) over the scattering angle in the top and bottom parts yields two scans over a single atom

$$
\begin{gathered}
\rho_{+}(R)=\int d q_{x} \int_{q_{y 1}}^{q_{y 2}} \rho(\mathbf{q}, \mathbf{q}) d q_{y}, \\
\rho_{-}(R)=\int d q_{x} \int_{-q_{y 2}}^{-q_{y 1}} \rho(\mathbf{q}, \mathbf{q}) d q_{y},
\end{gathered}
$$

where $q_{y}=k_{0} \theta$. This is shown in Fig. 4(c) for $\theta_{y 1}=20 \mathrm{mrad}$, $\theta_{y 2}=50 \mathrm{mrad}$. The difference in position between the maxima in the two scans is $\sim 0.06 \mathrm{~nm}$, indicating that Cs corrected machines and extreme stability are needed to see the effect. Even so, the signal will be very faint, such that noise will tend to override the effect. Dynamical diffraction of the incident and the outgoing electron on the lattice and remaining aberrations of the probe-forming lens and the spectrometer will also complicate the situation. Quantitative spin detection will therefore need elaborate calculations. 


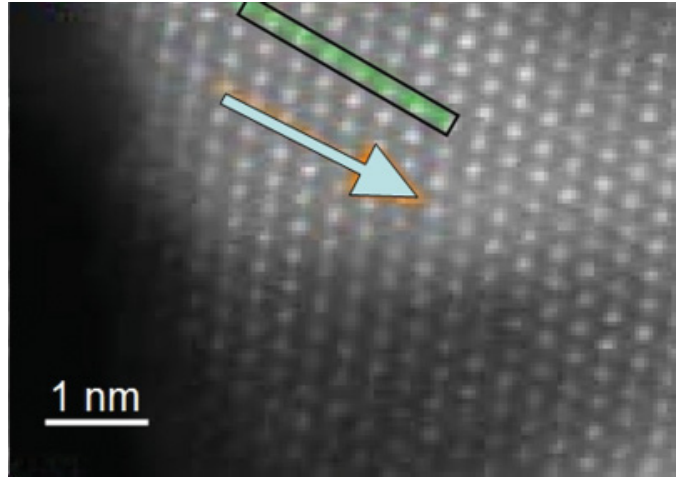

FIG. 5. (Color online) HAADF image of the border of the magnetite nanoparticle in the [111] zone axis orientation. The scan line is indicated as a box, the axis of energy dispersion (arrow) is almost perfectly parallel to the scan direction, thus $q_{y} \| y$. The bright dots are the A columns containing three Fe atoms per unit cell in this projection. Their projected distance is $0.33 \mathrm{~nm}$.

\section{EXPERIMENTAL EVIDENCE}

We have performed a feasibility experiment at the Daresbury SuperSTEM facility using a NION UltraSTEM100 microscope. ${ }^{34}$ The instrument is a dedicated, aberration corrected STEM operated at $100 \mathrm{kV}$ with a cold-FEG emitter. Its third-generation $\mathrm{C} 3 / \mathrm{C} 5$ aberration corrector allows a typical probe size of $0.1 \mathrm{~nm}$ at a beam current of $30 \mathrm{pA}$. Electron energy-loss spectroscopy (EELS) spectrum imaging at atomic resolution is done with a DigiScan2 scanning unit in combination with a Gatan Enfina spectrometer. The difficulty of the experiment stems from the extreme demand on stability, the rather long dwell times causing beam damage, remaining aberrations, and the low count rates. Spectra were collected during 0.2 s per position, corresponding to a dose of $\sim 5 \times 10^{7}$ electrons focused in the STEM spot.

We investigated a plateletlike magnetite nanoparticle of $\sim 15 \mathrm{~nm}$ diameter (Fig. 5). The thickness in this region was between 5 and $10 \mathrm{~nm}$, resembling as close as possible the single atomic row model. The STEM probe was scanned over a line of atoms marked in Fig. 5 as a rectangle. Magnetite is an inverted cubic spinel $X Y_{2} \mathrm{O}_{4}$ with $\mathrm{Fe}^{3+}$ ions at tetrahedral $X$ sites. The octahedral $Y$ sites are randomly occupied by $\mathrm{Fe}^{2+}$ and $\mathrm{Fe}^{3+}$ ions. $X$ and $Y$ sites are antiferromagnetically coupled. In (111) zone axis, there are two types of Fe columns (hereafter called $\mathrm{A}$ and $\mathrm{B})$. A contains two $X$ sites and one $Y$ site per elementary cell, B contains only one $Y$ site. The B columns are not visible in the high angle annular dark field (HAADF) image (Fig. 5) because of lower scattering strength and dynamical diffraction, confirmed by multislice simulations. However, very faint side maxima from the B columns can be seen in Fig. 6(a). Shown there are the top and bottom profiles of the scan integrated from 20 to 50 mrad after standard background subtraction, drift correction, and usual removal of the continuum signal beneath the white line. The post-edge continuum, often used in standard EMCD for normalizing, did not show periodic variations. Therefore, it was not necessary to correct for, the more so as this would have induced additional noise. A shift of the traces with respect to the atomic positions can already be guessed, although the noise is almost overriding the signal.
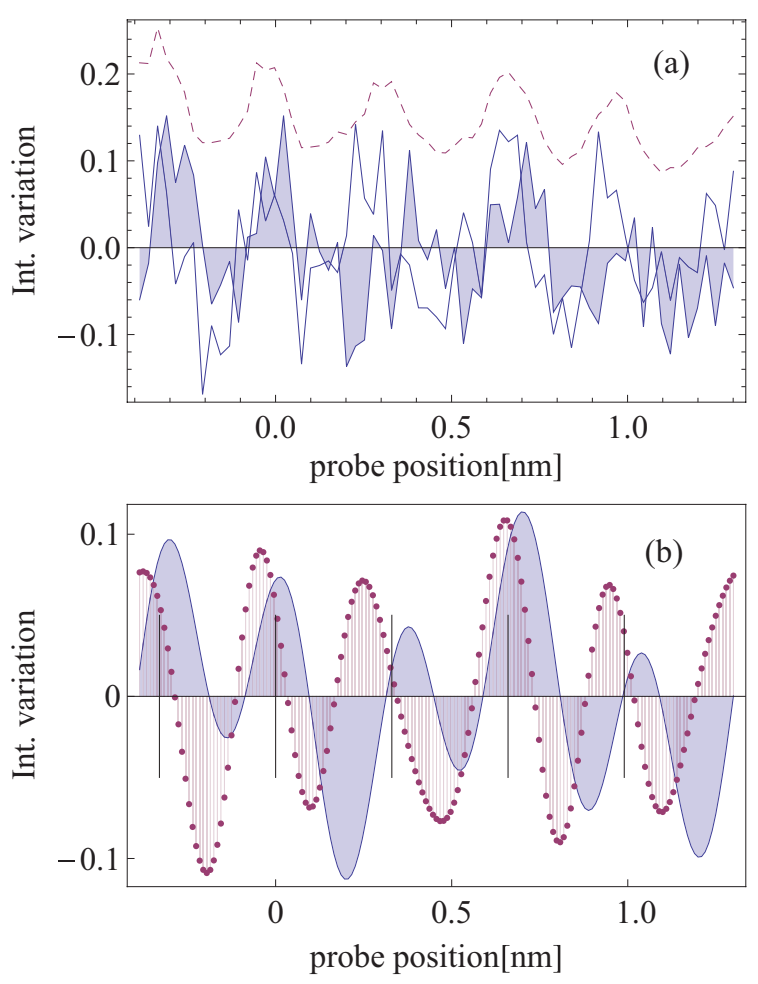

FIG. 6. (Color online) (a) Scan of the Fe $L_{3}$ white line signal after standard background subtraction, drift correction, and removal of the continuum. The filled curve shows the scan using the upper detector half (20 to $50 \mathrm{mrad}$ ), the empty curve is a scan using the lower detector half ( -50 to $-20 \mathrm{mrad})$. The HAADF signal is superposed (dashed) with maxima indicating the positions of the A columns. The B columns are visible as faint subsidiary peaks. (b) Same scans after Fourier filtering. Atom positions (HAADF maxima) are marked with vertical lines.

The number of electrons collected in the $L_{3}$ edge was $\sim 250$ in each half detector, causing a theoretical shot noise level of $3 \sigma=47$. Pre-edge extrapolation, instability, and other error sources add noise such that the $3 \sigma$ relative error amounts to $\sim 30 \%-40 \%$.

In order to demonstrate the predicted effect qualitatively, a Fourier analysis was performed on the two scans, retaining only coefficients up to lattice periodicity. The result is shown in Fig. 6(b). As predicted in Fig. 4, the maxima are shifted to both sides of the atom centers that are marked by vertical lines. The average distance between the top and bottom scan maxima is $0.076 \mathrm{~nm}$. One observes a rather large shift at the $0.33-\mathrm{nm}$ position that is caused by some irregularity (probably sudden drift). Excluding this value, the average difference between the respective maxima in the two scans is $0.65 \mathrm{~nm}$, in good agreement with the simulational result of $0.6 \mathrm{~nm}$ seen in Fig. 4(b). Despite the crude approximations and the simple model, the agreement is surprisingly good. More accurate models can be devised, but this is beyond the scope of this paper as noise, drift, and beam damage pose narrow limits on the interpretation of the data. More elaborate experiments with ultrathin magnetic specimens must be performed in order to confirm the present findings. 

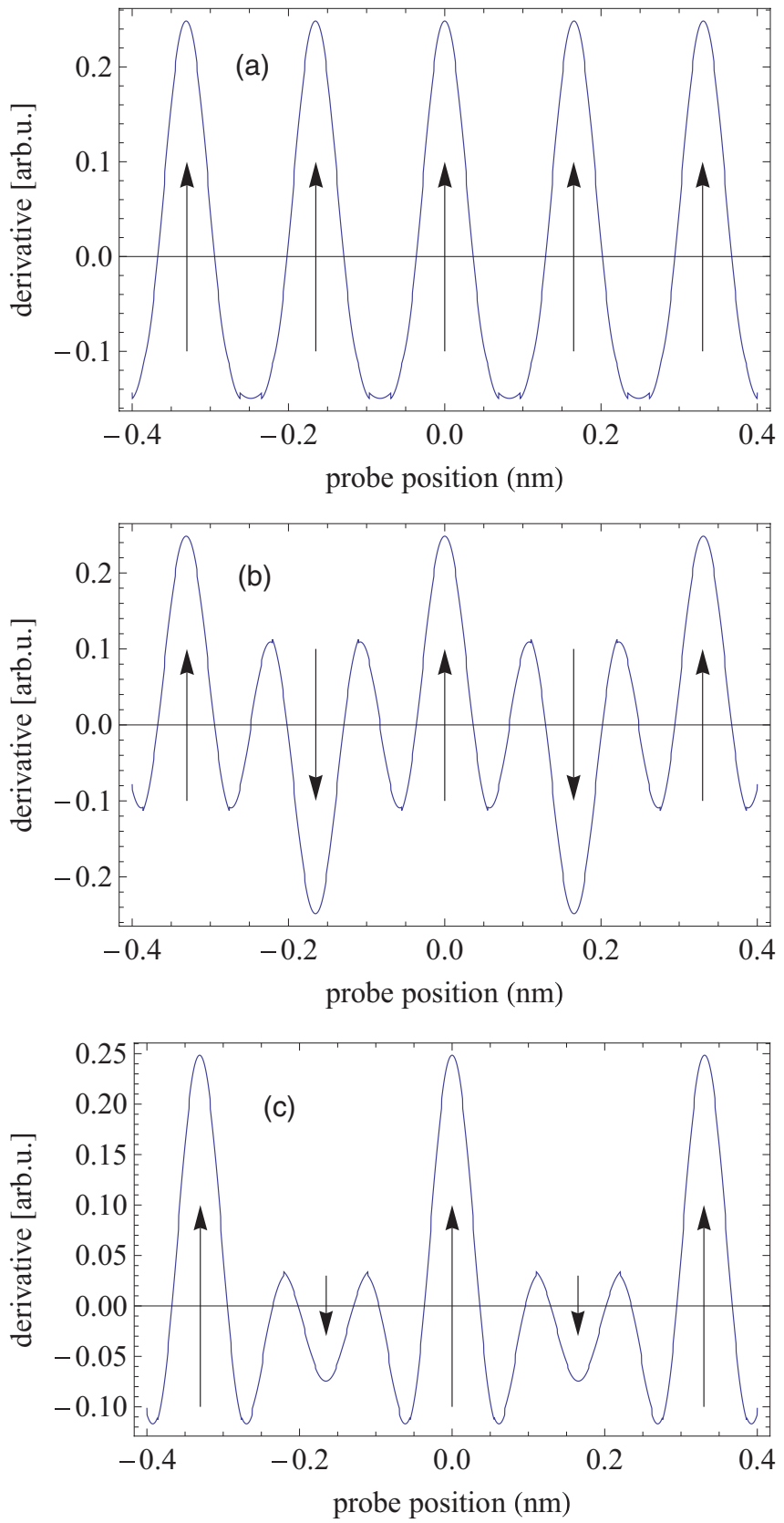

FIG. 7. (Color online) Hypothetical line scans showing the first derivative $d\left[\rho_{+}(R)-\rho_{-}(R)\right] / d r$ over an atomic row as in Fig. 5 for (a) ferromagnetic, (b) antiferromagnetic, and (c) ferrimagnetic ordering. (c) was calculated with a relative strength of $-30 \%$ for the spin-down moments, thus taking into account the strong channeling at the A columns.

\section{POTENTIAL APPLICATIONS}

\section{A. Detection of magnetic order}

As described above and shown in Fig. 4, the top/bottom asymmetry in the $\left(R, q_{y}\right)$ data set is caused by the spin polarization on the atomic site. That provides a method for spin mapping. A straightforward way to do so is to take the first derivative of the difference (top-bottom) line scans $\left(\rho_{+}^{\prime}-\rho_{-}^{\prime}\right)$. Since the slope of the difference signal is strongest at the atomic sites, this scan gives directly the sign and position of
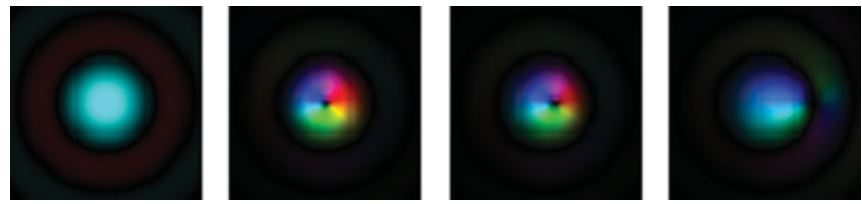

FIG. 8. (Color online) The phase and amplitude of the incident Airy disk of $0.1 \mathrm{~nm}$ diameter (left) and for the scattered wave from the $\mu=1$ transition. The atom is displaced from the beam center to the left by $0,0.01$, and $0.05 \mathrm{~nm}$. The images have a side length of $0.2 \mathrm{~nm}$.

the magnetic moment. Here, we assume a hypothetical system with the same lattice constant as magnetite, also in (111) zone axis orientation, to make connection to real systems. Figure 7 shows the derivative of $\left(\rho_{+}-\rho_{-}\right)$along a line scan as in Fig. 5 for different assumptions of the magnetic ordering (ferromagnetic, antiferromagnetic, and ferrimagnetic). To take account of the dechanneling and defocusing throughout the specimen, the data were convolved with a $0.1-\mathrm{nm}$ broadening function. The last panel resembles magnetite, in fact. In the (111) zone axis projection, the A columns contain two atoms spin up and on atom spin down, and the B columns contain one atom spin down, oriented along the $z$ axis in the magnetic field of the objective lens. On the other hand, the channeling is stronger on the deeper potential (A columns containing three atoms as compared to one on $\mathrm{B}$ ) so the beam will see more from the A columns than from the B columns. This will weigh the A columns stronger than the $\mathrm{B}$ ones. The exact weighting factor is impossible to obtain without solving the dynamical equation for the propagator in the lattice, but a weighting coefficient between $-50 \%$ and $-25 \%$ for the B columns is reasonable. Here, $-30 \%$ were assumed for the simulation. Theoretically, it should be possible to perform such scans not only along lines, but over areas. This should give atom-resolved maps of the element-specific magnetic moments and the magnetic ordering. But, before establishing such an analytical technique, the problems related to noise, stability, aberrations, and dynamical diffraction must be solved.

\section{B. Free electrons with angular momentum}

If an angstrom-sized spot is focused exactly at the center of an atom in a ferromagnet, the scattered electron has acquired orbital momentum. It is important to note that it is in a mixed state with contributions from the three transition channels $\mu \in[-1,1]$, each creating a pure vortex state with topological charge $\mu$. The expectation value of the angular momentum can
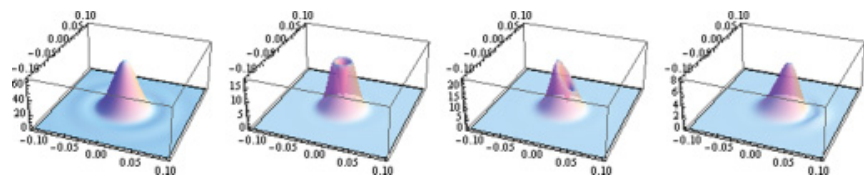

FIG. 9. (Color online) Intensity of the incident Airy disk (left) and $\operatorname{Tr}[\rho]$ for the scattered wave (mixed state from three transition channels) for atom displacements as in Fig. 8. 

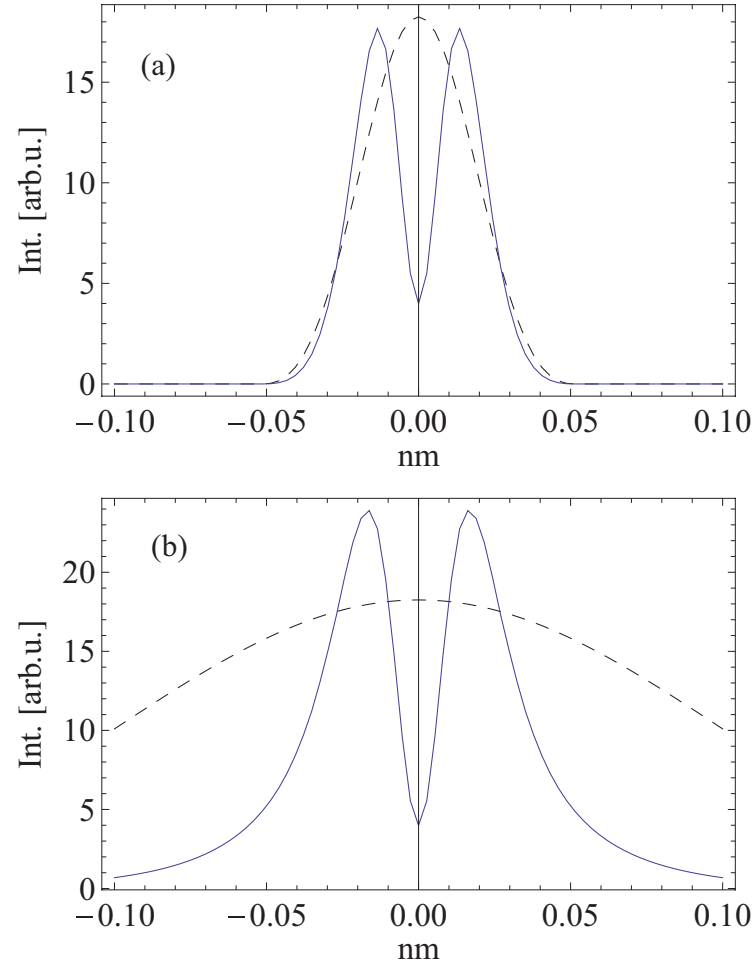

FIG. 10. (Color online) (a) Radial profiles of the incident 1- $\AA$ probe (dashed line) and the outgoing electron density of the mixed state when the atom is exactly centered on the beam. (b) The same for an incident probe of $5 \AA$ diameter.

be calculated from the coefficients $C_{j \mu}$ given in Eq. (6):

$$
\left\langle\mathcal{L}_{z}\right\rangle=\frac{\operatorname{Tr}_{\mu}\left[\mathcal{L}_{z} \rho\right]}{\operatorname{Tr}_{\mu}[\rho]} \in[-0.167 \hbar, 0.167 \hbar]
$$

depending on the spin polarization of the atom. This is a unique and simple method to create free electrons with orbital momentum, although the efficiency and the available momentum are probably too low to manipulate nanoparticles or even atoms by the torque. Another problem is the precise positioning of the probe on the atom. Figure 8 shows the phase and amplitude of the incident beam of $1 \AA$ diameter and the scattered beam for the $\mu=1$ transition when the atom is in the center, $0.01 \mathrm{~nm}$, and $0.05 \mathrm{~nm}$ sideways. The vortex structure disappears rapidly, also visible in the intensity distribution Fig. 9. Here, the total electron density $\operatorname{Tr}_{\mu}[\rho]$ is shown. The central dip, characteristic for the topological charge, remains at a deviation of $0.01 \mathrm{~nm}$, but has disappeared for $0.05 \mathrm{~nm}$.
Figure 10(a) compares the incident Airy disk with the radial profile of the outgoing mixed state. The scattered state is even narrower than the Airy disk. The high sensitivity of the outgoing vortex state to the probe position could be used for a more direct method of spin mapping with subatomic resolution than described above, e.g., with a vortex filter such as a holographic mask.

We simulated also the scattered state when the incident beam is broader $(0.5 \mathrm{~nm}$ diameter $)$. The result is shown in Fig. 10(b). The outgoing beam is broader than in case (a), but still much narrower than the incident Airy disk, and is again a superposition of electron vortices. The not so surprising consequence is that a thin ferromagnetic foil in an electron beam creates free electrons carrying angular momentum after energy filtering. This shows that vortices always have been there in EELS experiments on magnetic materials.

\section{CONCLUSION}

The coupling of an angstrom-sized electron probe to a spinpolarized transition creates a mixed state that contains electron vortices with nonzero orbital momentum. These states break the symmetry of the scattering distribution in the far field in a way characteristic for the chirality of the transition, a fact that can be used for the imaging of electron spins in real space with sub-angstrom resolution. A tentative experiment on a magnetite nanoparticle shows the expected asymmetry.

Apart from probing the local magnetic ordering, important for a number of technologically promising materials such as Heusler alloys, the proposed method bears promise for the mapping of spin polarizations of single atomic columns, be that in the vicinity of interfaces, magnetically dead layers, or magnetic core-shell structures. The creation of free electrons carrying angular momentum is theoretically feasible via spinpolarized electronic transitions. This works even for relatively broad incident beams passing a thin ferromagnetic foil.

\section{ACKNOWLEDGMENTS}

The authors thank A. Bleloch, S. Löffler, and P. Nellist for fruitful discussions and suggestions. P.S. acknowledges financial support from the Austrian Science Fund, Project No. I543-N20. The support of the EPSRC for the SuperSTEM facility is gratefully acknowledged. J.V. acknowledges support from the European Research Council under the 7th Framework Program (FP7), ERC Grant No. 246791-COUNTATOMS and ERC Starting Grant No. 278510-VORTEX.
${ }^{1}$ J. Verbeeck, H. Tian, and P. Schattschneider, Nature (London) 467, 301 (2010).

${ }^{2}$ J. Verbeeck, P. Schattschneider, S. Lazar, M. Stöger-Pollach, S. Löffler, A. Steiger-Thirsfeld, and G. Van Tendeloo, Appl. Phys. Lett. 99, 203109 (2011).

${ }^{3}$ P. Schattschneider, M. Stöger-Pollach, S. Löffler, A. Steiger-Thirsfeld, J. Hell, and J. Verbeeck, Ultramicroscopy 115, 21 (2012).
${ }^{4}$ J. Verbeeck, H. Tian, and A. Béché, Ultramicroscopy 113, 83 (2012).

${ }^{5}$ K. Y. Bliokh, Y. P. Bliokh, S. Savelev, and F. Nori, Phys. Rev. Lett. 99, 190404 (2007)

${ }^{6} \mathrm{P}$. Schattschneider and J. Verbeeck, Ultramicroscopy 111, 1461 (2011).

${ }^{7}$ K. Y. Bliokh, M. R. Dennis, and F. Nori, Phys. Rev. Lett. 107, 174802 (2011). 
${ }^{8}$ H. L. Xin and D. A. Muller, Nat. Nanotechnol. 5, 764 (2010).

${ }^{9}$ P. Schattschneider, S. Rubino, C. Hébert, J. Rusz, J. Kuneš, P. Novák, E. Carlino, M. Fabrizioli, G. Panaccione, and G. Rossi, Nature (London) 441, 486 (2006).

${ }^{10}$ S. Rubino, P. Schattschneider, M. Stöger-Pollach, C. Hébert, J. Rusz, L. Calmels, B. Warot-Fonrose, F. Houdellier, V. Serin, and P. Novak, J. Mater. Res. 23, 2582 (2008).

${ }^{11}$ P. Schattschneider, S. Rubino, M. Stöger-Pollach, C. Hébert, J. Rusz, L. Calmels, and E. Snoeck, J. Appl. Phys. 103, 07D931 (2008).

${ }^{12}$ P. Schattschneider, M. Stöger-Pollach, S. Rubino, M. Sperl, C. Hurm, J. Zweck, and J. Rusz, Phys. Rev. B 78, 104413 (2008).

${ }^{13}$ I. Ennen, S. Löffler, C. Kübel, A. Auge, A. Hütten, and P. Schattschneider, J. Magn. Magn. Mater. (in press, 2011).

${ }^{14}$ S. Y. Yu, Z. H. Liu, G. D. Liu, J. L. Chen, Z. X. Cao, G. H. Wu, B. Zhang, and X. X. Zhang, Appl. Phys. Lett. 89, 162503 (2006).

${ }^{15}$ P. J. Shamberger and F. S. Ohuchi, Phys. Rev. B 79, 144407 (2009).

${ }^{16}$ R. F. Klie, T. Yuan, M. Tanase, G. Yang, and Q. Ramasse, Appl. Phys. Lett. 96, 082510 (2010).

${ }^{17}$ P. D. Nellist, M. F. Chisholm, N. Dellby, O. L. Krivanek, M. F. Murfitt, Z. S. Szilagyi, A. R. Lupini, A. Borisevich, W. H. Sides Jr., and S. J. Pennycook, Science 305, 1741 (2004).

${ }^{18}$ O. L. Krivanek, M. F. Chisholm, V. Nicolosi, T. J. Pennycook, G. J. Corbin, N. Dellby, M. F. Murfitt, C. S. Own, Z. S. Szilagyi, M. P. Oxley, S. T. Pantelides, and S. J. Pennycook, Nature (London) 464, 571 (2010).

${ }^{19}$ D. A. Muller, Nat. Mater. 8, 263 (2009).
${ }^{20}$ Linear and Chiral Dichroism in the Electron Microscope, edited by P. Schattschneider (Pan Stanford Publishing, Singapore, 2012).

${ }^{21} \mathrm{P}$. Schattschneider, J. Verbeeck, and A. L. Hamon, Ultramicroscopy 109, 781 (2009).

${ }^{22} \mathrm{P}$. Schattschneider, J. Verbeeck, V. Mauchamp, M. Jaouen, and A. Hamon, Phys. Rev. B 82, 144418 (2010).

${ }^{23}$ S. D. Findlay, P. Schattschneider, and L. J. Allen, Ultramicroscopy 108, 58 (2007).

${ }^{24}$ Y. Kainuma, Acta Crystallogr. 8, 247 (1955).

${ }^{25}$ C. Dwyer, S. D. Findlay, and L. J. Allen, Phys. Rev. B 77, 184107 (2008).

${ }^{26} \mathrm{P}$. Schattschneider, M. Nelhiebel, and B. Jouffrey, Phys. Rev. B 59, 10959 (1999).

${ }^{27}$ L. J. Allen and T. W. Josefsson, Phys. Rev. B 52, 3184 (1995).

${ }^{28}$ J. Verbeeck, D. van Dyck, H. Lichte, P. Potapov, and P. Schattschneider, Ultramicroscopy 102, 239 (2005).

${ }^{29}$ B. T. Thole, P. Carra, F. Sette, and G. van der Laan, Phys. Rev. Lett. 68, 1943 (1992).

${ }^{30}$ J. Rusz, S. Rubino, and P. Schattschneider, Phys. Rev. B 75, 214425 (2007).

${ }^{31}$ J. Etheridge, S. Lazar, C. Dwyer, and G. A. Botton, Phys. Rev. Lett. 106, 160802 (2011).

${ }^{32}$ J. Rusz, O. Eriksson, P. Novák, and P. M. Oppeneer, Phys. Rev. B 76, 060408 (2007).

${ }^{33}$ L. Calmels, F. Houdellier, B. Warot-Fonrose, C. Gatel, M. J. Hytch, V. Serin, E. Snoeck, and P. Schattschneider, Phys. Rev. B 76, 060409(R) (2007).

${ }^{34}$ O. L. Krivanek, G. J. Corbin, N. Dellby, B. F. Elston, R. J. Keyse, M. F. Murfitt, C. S. Own, Z. S. Szilagyi, and J. W. Woodruff, Ultramicroscopy 108, 179 (2008). 